\title{
THE MOURRE ESTIMATE FOR DISPERSIVE $N$-BODY SCHRÖDINGER OPERATORS
}

\author{
JAN DEREZIŃSKI
}

\begin{abstract}
We prove the Mourre estimate for a certain class of dispersive $N$ body Schrödinger operators. Using this estimate we derive some properties of those operators such as the local finiteness of the finite spectrum and the absence of the singular continuous spectrum.
\end{abstract}

\section{INTRODUCTION}

Let $X$ be a vector space isomorphic to $\mathrm{R}^{n}$ and $H$ a selfadjoint operator on $L^{2}(X) . E_{\Delta}(H)$ will denote the spectral projection for $H$ onto the interval $\Delta \subset \mathbf{R}$ and $A=-\frac{i}{2}(\nabla \cdot x+x \cdot \nabla)$ will be the generator of dilations for $X$. We say that $H$ satisfies the Mourre estimate on $\Delta$ if and only if there exists a positive number $c$ and a compact operator $B$ such that

$$
E_{\Delta}(H) i[H, A] E_{\Delta}(H) \geq c E_{\Delta}(H)+B .
$$

It is well known that if $H$ is an $N$-body operator and $E_{0}$ is not its threshold then there exists a sufficiently small open interval $\Delta$ containing $E_{0}$ such that $H$ satisfies the Mourre estimate on $\Delta$. This result was first proved by $\mathrm{E}$. Mourre [M1] in the three-body case. The first proof for a large class of $N$-body Schrödinger operators was first given in [PSS]; then another, simpler proof was presented in [FH1]. The Mourre estimate has been successfully used to prove various facts about $N$-body Schrödinger operators including the absence of the singular continuous spectrum, the local $H$-smoothness of certain operators, the local finiteness of the point spectrum [M1, M2, PSS, CFKS, Ya], bounds on the exponential decay of the eigenfunctions, the nonexistence of positive eigenvalues [FH2] and a number of facts on the propagation of certain observables [SigSof1, SigSof2].

Regular $N$-body Schrödinger operators are very commonly used to describe the motion of $N$ interacting nonrelativistic particles. They are operators of the form

$$
H=\sum_{i=1}^{N} \frac{\left(D_{i}\right)^{2}}{2 m_{i}}+\sum_{i<j} V_{i j}\left(x_{i}-x_{j}\right)
$$

Received by the editors June 16, 1988.

1980 Mathematics Subject Classification (1985 Revision). Primary 47A10; Secondary 81F10. 
where $x_{i}$ is the position of the $i$ th particle, $m_{i}$ its mass, and $D_{i}$ the operator $i \nabla_{x_{i}}$ which describes the momentum of the $i$ th particle [RS3, RS4, Sig1, Ha].

In some situations we need to modify the kinetic energy in $H$ by replacing the momentum squared by certain more general differential operators.

For instance a system of $N$ interacting particles can be approximately described by the Hamiltonian

$$
H=\sum_{i=1}^{N} \sqrt{\left(D_{i}\right)^{2}+\left(m_{i}\right)^{2}}-m_{i}+\sum_{i<j} V_{i j}\left(x_{i}-x_{j}\right) .
$$

Another example of this sort arises if we consider a particle moving in a crystal. It is sometimes convenient to assume that its effective kinetic energy is not the momentum squared but a fairly arbitrary function $\omega_{i}$ of the momentum. If we make this assumption then a system of $N$ interacting particles in a crystal will be described by a Hamiltonian of the form

$$
H=\sum_{i=1}^{N} \omega_{i}\left(D_{i}\right)+\sum_{i<j} V_{i j}\left(x_{i}-x_{j}\right) .
$$

Hamiltonians similar to (1.3) are studied also in the context of a system of $N$ interacting spins on a lattice.

All the above-mentioned operators fall into the class of operators which we will call the dispersive $N$-body Schrödinger operators. They are selfadjoint operators on $L^{2}(X)$ of the form

$$
H=\omega(D)+\sum_{a \in \mathscr{A}} v_{a}\left(\pi^{a} x\right)
$$

where $\left\{X_{a}: a \in \mathscr{A}\right\}$ is a family of subspaces of $X, X^{a}=X / X_{a}, \pi^{a}$ are the canonical surjections from $X$ onto $X^{a}, v^{a}$ are real functions on $X^{a}, K$ is the space dual to $X, \omega$ is a real function on $K$ and $D$ is the operator $i \nabla$ on $L^{2}(X)$.

An important subclass of the dispersive $N$-body Schrödinger operators is obtained if we set $\omega(k)=k^{2}$. Those operators will be called in our paper the nondispersive $N$-body Schrödinger operators. They are a natural generalization of the operators of the form (1.1) and have been studied for instance in [FH1, FH2, A]. (Note that if $\omega(k)$ is a positive definite quadratic form on $K$ then by an appropriate change of coordinates in the configuration space we can transform $H$ into a nondispersive $N$-body Schrödinger operator.)

This paper is devoted to a proof of certain properties of a class of dispersive $N$-body Schrödinger operators. Let us begin the description of our results with a few words on the hypotheses needed in our paper.

Those hypotheses fall into two categories: the explicit and the implicit ones. The explicit assumptions that we impose on $\omega$ mean roughly that $\omega$ is nonnegative, differentiable and goes to infinity as $|k|$ goes to infinity. The explicit assumptions on the potentials are quite straightforward generalizations of the 
analogous assumptions contained in [FH1 or PSS] needed in the proof of the Mourre estimate in the nondispersive case. Roughly speaking they mean that $v_{a}\left(x^{a}\right)$ goes to zero if $\left|x^{a}\right|$ goes to infinity.

Unfortunately we also will need certain implicit hypotheses on $H$. Those hypotheses describe the spectral properties of the so-called cluster Hamiltonians $H_{a}$ which are naturally associated with the operator $H$. To explain the necessity of those hypotheses it is helpful to take a look at the nondispersive case. In this case the cluster Hamiltonians $H_{a}$ can be written as a sum of a Hamiltonian $H^{a}$ that describes the intracluster energy and a term that describes the kinetic energy of the intercluster motion. In the dispersive case this is no longer possible in general. All we can do is write $H_{a}$ as a direct integral with fibers labelled by $K_{a}$ (the space dual to $X_{a}$ ). The projections onto the pure point spectrum of the fibers of those integrals do not depend on $k_{a} \in K_{a}$ in the nondispersive case, but in the dispersive case they may depend on $k_{a}$ in a way that is difficult to control. We deal with this problem by assuming that the cluster Hamiltonians have especially nice properties around a certain fixed number $E_{0}$.

We prove that if all the above mentioned hypotheses are true then there exists an interval $\Delta$ containing $E_{0}$ such that operators $H$ of the form (1.1) satisfy the Mourre estimate on $\Delta$. Our proof closely follows the proof of the Mourre estimate for nondispersive $N$-body Schrödinger Hamiltonians contained in [FH1]. In spite of its similarity though, there are a number of technical problems that do not exist in the nondispersive case.

Once we have proved the Mourre estimate we can show various interesting results about the spectral properties of dispersive $N$-body Schrödinger operators almost without any work. We just need to invoke certain abstract theorems contained in [M1, M2, PSS, CFKS] due to E. Mourre and improved later on by P. Perry, I. M. Sigal and B. Simon. We show in particular that if $H$ satisfies certain hypotheses of the type mentioned above then there exists an open interval $\Delta$ containing $E_{0}$ such that:

(1) the pure point spectrum of $H$ in $\Delta$ is finite;

(2) there is no singular continuous spectrum in $\Delta$;

(3) the operator $(|x|+1)^{-1 / 2-\varepsilon}$ is locally $H$-smooth on $\Delta$ (see [Ka1, Ka2, RS4] for the definition of $H$-smooth operators).

Let us make a comment on result (3) mentioned above. The results of this kind are very useful in the scattering theory. For instance, the local $\mathrm{H}$ smoothness of $(|x|+1)^{-1 / 2-\varepsilon}$ in the nondispersive case is an important tool in the proof of the asymptotic completeness of the $N$-body scattering in [SigSof 1] (see also [De1]). We use (3) in [De2] where we study the local Kato-smoothness of various pseudodifferential operators with respect to certain dispersive $\mathrm{N}$ body Schrödinger operators.

Unfortunately, we think that it can be quite difficult to verify the implicit hypotheses on $H$ used in our paper in concrete cases. The only case where we know how to check those hypotheses is the case of $\omega(k)$ being a positive 
definite quadratic form (which as we mentioned earlier can be reduced to the nondispersive case). In this case our implicit hypotheses on $H$ are satisfied if we assume that $E_{0}$ is not a threshold of $H$. (Note that in the general dispersive case we are not even able to define the notion of a threshold. In the nondispersive case, on the other hand, the set of thresholds of a given Hamiltonian can be shown to be a relatively small set, namely, a closed countable subset of the real numbers-see [M1, PSS].)

\section{BASIC NOTATION}

Throughout this paper $X$ will denote a vector space isomorphic to $\mathbb{R}^{n}$. $\left\{X_{a}: a \in \mathscr{A}\right\}$ will be a certain finite family of its subspaces. To be consistent with the notation used in the literature we will write $a_{1} \subset a_{2}$ whenever $X_{a_{1}} \supset X_{a_{2}}$ and $a_{1} \cup a_{2}=a_{3}$ whenever $X_{a_{1}} \cap X_{a_{2}}=X_{a_{3}} \cdot X_{a_{\min }}$ will denote $X$ and $X_{a_{\max }}=\{0\}$. We will assume the following properties of $\mathscr{A}$ :

(1) $a_{\min } \in \mathscr{A}$

(2) $a_{\max } \in \mathscr{A}$,

(3) if $a_{1}, a_{2} \in \mathscr{A}$ then $a_{1} \cup a_{2} \in \mathscr{A}$.

$\# a$ will denote the maximal number of distinct $a_{i}$ 's such that $a=a_{n} \varsubsetneqq$ $a_{n-1} \varsubsetneqq \cdots \varsubsetneqq a_{1}=a_{\max }$. Note that in the usual many particle case the set $\mathscr{A}$ corresponds to the set of various cluster decompositions and \#a has the meaning of the numbers of clusters in a given cluster decomposition.

We denote $X / X_{a}$ by $X^{a}$. The spaces dual to $X_{a}, X$ and $X^{a}$ will be denoted by $K_{a}, K$ and $K^{a}$. We will fix a scalar product in $X$ which will enable us to identify $X^{a}$ with a certain subspace of $X$ and $K_{a}$ with a certain subspace of $K$. $\pi_{a}$ and $\pi^{a}$ will denote the projections of $K$ and $X$ onto $K_{a}$ and $X^{a}$ respectively. If $b \subset a$ then $X_{a} / X_{b}$ will be denoted by $X_{a}^{b}$.

If $y \in X$ and $r \geq 0$ then $B(y, r)$ denotes $\{x \in X:|x-y| \leq r\}$.

$D$ will denote the operator $-i \nabla$ and $D_{a}=-i \nabla_{a}$.

$\alpha$ will be the generic name of a multi-index.

If $\mathscr{H}$ is a Hilbert space then $B(\mathscr{H})$ denotes the set of bounded operators on $\mathscr{H}$. If $B$ is a selfadjoint operator then $\sigma(B)$ denotes its spectrum. Similarly, $\sigma_{\mathrm{c}}(B), \sigma_{\mathrm{ac}}(B), \sigma_{\mathrm{sc}}(B), \sigma_{\mathrm{disc}}(B)$ and $\sigma_{\mathrm{ess}}(B)$ denote its continuous, absolutely continuous, singular continuous, discrete and essential spectra. If $\Delta$ is a measurable subset of $\mathbb{R}$ then $E_{\Delta}(B)$ denotes the spectral projection of $B$ onto $\Delta$. Similarly, $E_{\Delta}^{\mathrm{c}}(B), E_{\Delta}^{\mathrm{ac}}(B)$, etc. denote the spectral projections of $B$ onto the continuous, absolutely continuous, etc. part of the spectrum contained in $\Delta$.

If $y \in X$ then we define the unitary operator $U_{y}$ on $L^{2}(X)$ such that $\left(U_{y} \varphi\right)(x)=\varphi(x-y)$. An operator $B \in B\left(L^{2}(X)\right)$ will be called $a$-fibered whenever $U_{y} B=B U_{y}$ for all $y \in X_{a}$. Such operators can be decomposed as $B=\int_{K_{a}}^{\oplus} d k_{a} B\left(k_{a}\right)$ where $k_{a} \mapsto B\left(k_{a}\right)$ is a function from $L^{\infty}\left(K_{a}, B\left(L^{2}\left(X^{a}\right)\right)\right)$. Note that $\|B\|=\operatorname{esssup}\left\|B\left(k_{a}\right)\right\|$. We will say that $B$ is $a$-continuous if and only 
if it is bounded, $a$-fibered and the function $k_{a} \mapsto B\left(k_{a}\right)$ is norm-continuous. We will say that $B$ is $a$-compact if it is $a$-continuous, $B\left(k_{a}\right)$ is compact on $L^{2}\left(X^{a}\right)$ and $\lim _{\left|k_{a}\right| \rightarrow \infty}\left\|B\left(k_{a}\right)\right\|=0$. We will say that $B$ is $a$-finite if it is a finite linear combination of operators of the form $\operatorname{Pf}\left(D_{a}\right)$ where $P$ is a finite rank operator on $L^{2}\left(X^{a}\right)$ and $f \in C_{0}^{\infty}\left(K_{a}\right)$.

The above defined classes of operator were used in [PSS]. The proofs of the following simple properties of those operators can be found in [PSS].

Lemma 2.1. (a) The set of a-compact operators is norm-closed.

(b) If $C$ is a-compact and $B$ is a-continuous then $C B$ is a-compact.

(c) The set of a-finite operators is dense in the set of a-compact operators.

(d) If $C$ is a-compact, $B_{n} \in L^{2}\left(X^{a}\right)$ for $n \in \mathbf{N}$ and $\mathrm{s}-\lim _{n \rightarrow \infty} B_{n}=0$ then $\lim _{n \rightarrow \infty}\left\|B_{n} C\right\|=0$.

Let us prove yet another property of $a$-compact operators.

Lemma 2.2. Let $C$ be a-compact and $B_{n}$ be a-continuous for $n \in \mathbb{N}$. Suppose that $k_{a} \in K_{a}$ and $\lim _{n \rightarrow \infty} B_{n}\left(k_{a}\right)=0$. Then for every $\varepsilon>0$ there exists $\delta>0$ and $n \in \mathbb{N}$ such that

$$
\left\|E_{B\left(k_{a}, \delta\right)}\left(D_{a}\right) B_{n} C\right\| \leq \varepsilon .
$$

Proof. It is enough to assume that $C=P_{\varphi} f\left(D_{a}\right)$ where $f \in C_{0}^{\infty}\left(K_{a}\right), \varphi \in$ $L^{2}\left(X^{a}\right),\|\varphi\|=1$ and $P_{\varphi}$ is the projection onto the subspace spanned by $\varphi$. Then

$$
\left\|E_{B\left(k_{a}, \delta\right)}\left(D_{a}\right) B_{n} C\right\|=\sup _{p_{a} \in B\left(k_{a}, \delta\right)}\left\|B_{n}\left(p_{a}\right) f\left(p_{a}\right) \varphi\right\| .
$$

We will find an $n \in \mathbb{N}$ such that

$$
\left\|B_{n}\left(k_{a}\right) f\left(k_{a}\right) \varphi\right\|<\varepsilon / 2 .
$$

We will also find $\delta>0$ such that

$$
\sup _{p_{a} \in B\left(k_{a}, \delta\right)}\left\|B_{n}\left(p_{a}\right) f\left(p_{a}\right) \varphi-B_{n}\left(k_{a}\right) f\left(k_{a}\right) \varphi\right\|<\varepsilon / 2 .
$$

Now (2.2)-(2.4) imply (2.1). Q.E.D.

\section{The Hamiltonian}

In this section we introduce the assumptions on the Hamiltonian that we will use in our paper and study their most immediate consequences.

Let $\omega$ be a real infinitely differentiable nonnegative function on $K$ such that $\lim _{|k| \rightarrow \infty} \omega(k)=\infty$. For every $a \in \mathscr{A}$ let $v_{a}$ be a real-valued measurable function on $X^{a}$ such that the operator

$$
v_{a}\left(\pi^{a} x\right)(\omega(D)+1)^{-1}
$$


is a-compact. (For example $v_{a}$ can be a bounded measurable function on $X^{a}$ such that $\lim _{\left|x^{a}\right| \rightarrow \infty} v_{a}\left(x^{a}\right)=0$.) Clearly, $v_{a}\left(\pi^{a} x\right)$ 's are relatively bounded perturbations of $\omega(D)$ with an infinitesimal bound.

Let us set

$$
V(x)=\sum_{a \in \mathscr{A}} v_{a}\left(\pi^{a} x\right)
$$

We define $H$ to be the selfadjoint operator on $L^{2}(X)$ such that $\mathscr{D}(H)=$ $\mathscr{D}(\omega(D))$ and $H=\omega(D)+V(x)$.

Define also

$$
V_{a}(x)=\sum_{b \subset a} v_{b}\left(\pi^{b} x\right)
$$

and

$$
I_{a}(x)=\sum_{b \not \subset a} v_{b}\left(\pi^{b} x\right)
$$

The so-called "cluster Hamiltonians" $H_{a}$ are defined as selfadjoint operators such that $\mathscr{D}\left(H_{a}\right)=\mathscr{D}(\omega(D))$ and $H_{a}=\omega(D)+V_{a}(x)$.

Note that $H_{a_{\min }}=\omega(D)$ and $H_{a_{\max }}=H$. We will often write $H_{0}$ instead of $\omega(D)$. Note also that if $b \subset a$ then $H_{b}$ is $a$-fibered and we can write

$$
H_{b}=\int_{K_{a}}^{\oplus} d k_{a} H_{b}\left(k_{a}\right) .
$$

Let $E \in \mathbb{R}$. Then we define $E S_{a}(E)=\left\{k_{a} \in K_{a}: E \in \sigma\left(H_{a}\left(k_{a}\right)\right)\right\}, E S_{a}^{p p}(E)$ $=\left\{k_{a} \in K_{a}: E \in \sigma_{p p}\left(H_{a}\left(k_{a}\right)\right)\right\}$, etc.

Now let us give a list of hypotheses to which we will refer in this paper. The first family of hypotheses deals with the kinetic energy term.

A1. For all $\alpha$

$$
\lim _{|k| \rightarrow \infty} \frac{\left|\partial_{k}^{\alpha} \omega(k)\right|}{\omega(k)+1}=0
$$

$\mathrm{A}^{\prime}$. For all $\alpha$

$$
\frac{\left|\partial_{k}^{\alpha} \omega(k)\right|}{\omega(k)+1} \leq c_{\alpha} .
$$

A2. There exist $c$ and $N$ such that

$$
\omega(k) \leq c\left(\left|k-k^{\prime}\right|+1\right)^{N} \omega\left(k^{\prime}\right) .
$$

A3. For all $\alpha$

$$
\lim _{|k| \rightarrow \infty} \frac{\left|\partial_{k}^{\alpha}(k \cdot \nabla \omega(k))\right|}{\omega(k)+1}=0 .
$$

A4.

$$
\frac{\left|(k \cdot \nabla)^{2} \omega(k)\right|}{(\omega(k)+1)^{2}} \leq c .
$$

A5.

$$
\frac{|k|}{(\omega(k)+1)} \leq c .
$$


Note that $\mathrm{Al}^{\prime}$ is weaker than $\mathrm{A} 1$. Next we list the assumptions concerning the potentials.

B1. The form

$$
(\omega(D)+1)^{-1} \pi^{a} x \cdot \nabla v_{a}\left(\pi^{a} x\right)(\omega(D)+1)^{-1}
$$

extends from the space of Schwartz test functions $\mathscr{S}(X)$ to an a-compact operator.

$\mathrm{B1}^{\prime}$. The form

$$
(\omega(D)+1)^{-1} \pi^{a} x \cdot \nabla v_{a}\left(\pi^{a} x\right)(\omega(D)+1)^{-1 / 2}
$$

extends to a bounded operator.

B2. The form

$$
(\omega(D)+1)^{-1}\left(\pi^{a} x \cdot \nabla\right)^{2} v_{a}\left(\pi^{a} x\right)(\omega(D)+1)^{-1}
$$

extends to a bounded operator.

Note that hypotheses $\mathrm{B} 1, \mathrm{~B} 1^{\prime}$ and $\mathrm{B} 2$ are close analogs of similar hypotheses found in [PSS, FH1, CFKS].

Now we would like to state two implicit hypotheses that will play an important role in our paper. They describe the spectral behavior of the cluster Hamiltonians in a vicinity of a certain energy $E_{0}$ that we keep fixed throughout the paper.

$\mathrm{C} 1$. For every $k_{a} \in E S_{a}^{p p}\left(E_{0}\right)$ there exists an open set $U_{k_{a}}$ in $K_{a}$ containing $k_{a}$, a $C^{1}$-function $U_{k_{a}} \ni p_{a} \mapsto \eta_{k_{a}}\left(p_{a}\right) \in \mathbf{R}$ and a norm-continuous function

$$
U_{k_{a}} \ni p_{a} \mapsto P_{k_{a}}\left(p_{a}\right) \in B\left(L^{2}\left(X^{a}\right)\right)
$$

with values in orthogonal projections such that

$$
P_{k_{a}}\left(k_{a}\right)=E_{\left\{E_{0}\right\}}^{p p}\left(H\left(k_{a}\right)\right)
$$

and

$$
H_{a}\left(p_{a}\right) P_{k_{a}}\left(p_{a}\right)=\eta_{k_{a}}\left(p_{a}\right) P_{k_{a}}\left(p_{a}\right) .
$$
then

C2. There exists a positive number $c_{0}$ such that if $a \in A$ and $k_{a} \in E S_{a}^{p p}\left(E_{0}\right)$

$$
k_{a} \cdot \nabla \eta_{k_{a}}\left(k_{a}\right) \geq c_{0}
$$

Let us look at the meaning of the above hypotheses if $\omega(k)=k^{2}$. Clearly, hypotheses A1-A5 are satisfied in this case. The a-compactness of (3.1) and hypothesis B1 are equivalent to the compactness of

$$
v_{a}\left(x^{a}\right)\left(-\Delta^{a}+1\right)^{-1}
$$

and

$$
\left(-\Delta^{a}+1\right)^{-1} x^{a} \nabla v_{a}\left(x^{a}\right)\left(-\Delta^{a}+1\right)^{-1}
$$

on $L^{2}\left(X^{a}\right)$. Note that identical assumptions on the potentials are contained in [FH1]. Hypotheses B2 and B3 are closely related to hypotheses 2 and 3 from 
Chapter 4 of [CFKS]. Hypothesis $\mathrm{C} 1$ is satisfied in the nondispersive case for all values of $E_{0}$. To see this let us note that in the case $H_{a}\left(k_{a}\right)=\left(k_{a}\right)^{2}+H^{a}$ where $H^{a}$ is a certain operator on $L^{2}\left(X^{a}\right)$ that does not depend on $k_{a}$. Let $\mathscr{T}_{a}$ denote the set of eigenvalues of $H^{a}$ (which are usually called the thresholds of $H$ corresponding to $a \in \mathscr{A})$. Then $E S_{a}^{p p}(E)=\left\{k_{a} \in K_{a}: E-k_{a}^{2} \in \mathscr{T}_{a}\right\}$. Now if $k_{a} \in E S_{a}^{p p}\left(E_{0}\right)$ then we can set $U_{k_{a}}=K_{a}, \eta_{k_{a}}\left(p_{a}\right)=\left(p_{a}\right)^{2}+E_{0}-\left(k_{a}\right)^{2}$ and $P_{k_{a}}\left(p_{a}\right)=E_{\left\{E_{0}-\left(k_{a}\right)^{2}\right\}}^{p p}\left(H^{a}\right)$. We easily compute that $k_{a} \nabla \eta_{k_{a}}\left(k_{a}\right)=2\left(k_{a}\right)^{2}$. Thus in the nondispersive case C2 is satisfied if and only if $E_{0} \notin \bigcup_{a \neq a_{\max }} \mathscr{T}_{a}$ (in other words, if $E_{0}$ is not a threshold of $H$ ).

At the end of this section we state a number of propositions about basic properties of $H_{a}$. They are straightforward generalizations of similar facts which are true in the nondispersive case. For example, it is easy to show the following fact.

Lemma 3.1. Assume $\mathrm{Al}^{\prime}$. Let $b \subset a$ and let $z$ belong to the resolvent set of $H_{b}\left(k_{a}\right)$. Then the function $K_{a} \ni k_{a} \mapsto\left(H_{b}\left(k_{a}\right)-z\right)^{-1} \in B\left(L^{2}\left(X^{a}\right)\right)$ is normdifferentiable.

By standard methods contained e.g. in [RS4 or $\mathrm{Ka} 3$ ] the above lemma implies the following corollary.

Corollary 3.2. Assume $\mathrm{Al}^{\prime}$. Let $k_{a} \in E S_{a}^{\mathrm{disc}}\left(E_{0}\right)$. Then the following holds:

(a) There exists $\delta>0$, an open set $U$ in $K_{a}$ containing $k_{a}$ and an infinitely norm-differentiable function $U \ni p_{a} \mapsto P\left(p_{a}\right) \in B\left(L^{2}\left(X^{a}\right)\right)$ with values in orthogonal finite-dimensional projections such that

$$
P\left(p_{a}\right)=E_{\left[E_{0}-\delta, E_{0}+\delta\right]}\left(H_{a}\left(p_{a}\right)\right)
$$

for $p_{a} \in U$ and

$$
P\left(k_{a}\right)=E_{\left\{E_{0}\right\}}\left(H_{a}\left(k_{a}\right)\right) .
$$

(b) If $U \ni p_{a} \mapsto \eta\left(p_{a}\right) \in \mathbb{R}$ is a function such that $H_{a}\left(p_{a}\right) P\left(p_{a}\right)=\eta\left(p_{a}\right) P\left(p_{a}\right)$ then $\eta\left(p_{a}\right)$ is infinitely differentiable.

(c) If $\operatorname{dim} E_{\left\{E_{0}\right\}}\left(H\left(k_{a}\right)\right)=1$ then there exists an open set $\tilde{U}$ in $K_{a}$ containing $k_{a}$ and an infinitely differentiable function $\tilde{U} \ni p_{a} \rightarrow \eta\left(p_{a}\right) \in \mathbb{R}$ such that $H_{a}\left(p_{a}\right) P\left(p_{a}\right)=\eta\left(p_{a}\right) P\left(p_{a}\right)$.

If we compare Corollary 3.2 with hypothesis $\mathrm{C} 1$ we see that in some cases $\mathrm{C} 1$ is satisfied automatically. This happens in particular if $E_{0}$ is a nondegenerate isolated eigenvalue for all $a \in A$ and $k_{a} \in E S_{a}^{p p}\left(E_{0}\right)$. Unfortunately, if $E_{0}$ is an isolated degenerate eigenvalue of $H_{a}\left(k_{a}\right)$ then it may be a point of bifurcation of the eigenvalues of the family $p_{a} \mapsto H_{a}\left(p_{a}\right)$. If $E_{0}$ is an imbedded eigenvalue of $H_{a}\left(k_{a}\right)$ then in general we can say nothing about the point spectrum of $H_{a}\left(p_{a}\right)$ for $p_{a}$ close to $k_{a}$.

The proof of the next fact is also left to the reader. 
Proposition 3.3. Let $b \subset a$. Then $\lim _{\left|k_{a}\right| \rightarrow \infty} \inf \left(\sigma\left(H_{b}\left(k_{a}\right)\right)\right)=\infty$.

Finally let us state a version of the HVZ theorem (see e.g. [RS4]).

Proposition 3.4. Assume $\mathrm{A} 1$ and $\mathrm{A} 2$. Then

$$
\sigma_{\text {ess }}\left(H_{a}\left(k_{a}\right)\right)=\bigcup_{\substack{b \subset a \\ \# b=\# a+1}} \sigma\left(H_{b}\left(k_{a}\right)\right) .
$$

\section{VIRIAL THEOREM}

The virial theorem says that if $\psi$ is an eigenfunction of a Schrödinger operator $H$ then $(\psi, i[H, A] \psi)=0$. This theorem is formally obvious but since $H$ and $A$ may be unbounded some care is required in its proof. Its proofs, under various assumptions on $H$, can be found in [Kalf, We, PSS, CFKS]. The virial theorem is an important ingredient of the proof of the Mourre estimate [M1, FH1, PSS]. We will need a slightly modified variant of this theorem which is better suited to studying $a$-fibered operators.

In this section we assume hypothesis B1. It guarantees that $i[H, A]$ and $i\left[H_{a}, A\right]$ can be defined as quadratic forms on $\mathscr{D}\left(H_{0}\right)$. Note that $i\left[H_{a} A\right]$ is $a$-fibered-in fact this is one of the main reasons why the generator of dilations $A$ is so useful in the $N$-body quantum mechanics. It is also easy to see that the functions

$$
k_{a} \mapsto\left(H_{0}\left(k_{a}\right)+1\right)^{-1} i\left[H_{a}, A\right]\left(k_{a}\right)\left(H_{0}\left(k_{a}\right)+1\right)^{-1}
$$

are infinitely norm-differentiable.

Let us state our modification of the virial theorem. Note that (b) is the traditional virial theorem.

Theorem 4.1. Let $a \in \mathscr{A}$. Suppose that $Q_{1}$ and $Q_{2}$ are a-fibered bounded operators whose ranges are contained in $\mathscr{D}\left(H_{0}\right)$.

(a) If $a \neq a_{\max }$ assume that

$$
H_{a} Q_{i}=\int_{K_{a}}^{\oplus} d k_{a} \eta\left(k_{a}\right) Q_{i}\left(k_{a}\right)
$$

for $i=1,2$ and for some bounded $C^{1}$-function $\eta$. Then

$$
Q_{1} i\left[H_{a}, A\right] Q_{2}=Q_{1} D_{a} \cdot \nabla \eta\left(D_{a}\right) Q_{2} .
$$
$=0$.

(b) If $a=a_{\max }$ we assume that $H Q_{i}=E Q_{i}$ for $i=1,2$. Then $Q_{1} i[H, A] Q_{2}$

In the proof of this theorem we need the following technical lemma which is proved in [CFKS, Lemma 4.5].

Lemma 4.2. Let $R_{\lambda}=i \lambda(A+i \lambda)^{-1}$. Then for big $\lambda\left(H_{0}+1\right) R_{\lambda}\left(H_{0}+1\right)^{-1}$ is bounded and $\lim _{\lambda \rightarrow \infty}\left(H_{0}+1\right) R_{\lambda}\left(H_{0}+1\right)^{-1}=1$.

Proof of Theorem 4.1. We will show only (a). We will follow closely the approach of [PSS, CFKS]. Define $A_{\lambda}=A R_{\lambda}$. Then as a form on $\mathscr{D}\left(H_{0}\right)$ we have 
$i\left[H_{a}, A_{\lambda}\right]=R_{\lambda} i\left[H_{a}, A\right] R_{\lambda}$. Thus if $\varphi_{1}, \varphi_{2} \in \mathscr{D}\left(H_{0}\right)$ then

$$
\left(\varphi_{1}, i\left[H_{a}, A_{\lambda}\right] \varphi_{2}\right) \underset{\lambda \rightarrow \infty}{\longrightarrow}\left(\varphi_{1}, i\left[H_{a}, A\right] \varphi_{2}\right) .
$$

On the other hand if $\psi_{1}, \psi_{2} \in L^{2}(X)$ then

$$
\begin{aligned}
\left(Q_{1} \psi_{1}, i\left[H_{a}, A_{\lambda}\right] Q_{2} \psi_{2}\right) & =\left(Q_{1} \psi_{1}, i\left[\eta\left(D_{a}\right), A_{\lambda}\right] Q_{2} \psi_{2}\right) \\
& =\left(Q_{1} \psi_{1}, R_{\lambda} i\left[\eta\left(D_{a}\right), A\right] R_{\lambda} Q_{2} \psi_{2}\right) \\
& =\left(R_{\lambda}^{*} Q_{1} \psi_{1}, D_{a} \cdot \nabla \eta\left(D_{a}\right) R_{\lambda} Q_{2} \psi_{2}\right) \\
& \underset{\lambda \rightarrow \infty}{\longrightarrow}\left(Q_{1} \psi_{1}, D_{a} \cdot \nabla \eta\left(D_{a}\right) Q_{2} \psi_{2}\right) . \quad \text { Q.E.D. }
\end{aligned}
$$

\section{MourRe estimate}

The main intermediate result of our paper is the following theorem:

Theorem 5.1. Assume $\mathrm{A} 1, \mathrm{~A} 2, \mathrm{~A} 3, \mathrm{~B} 1, \mathrm{C} 1$, and $\mathrm{C} 2$ to be true. Then there exists an open interval $\Delta$ containing $E_{0}$ such that $H$ satisfies the Mourre estimate on $\Delta$.

We also would like to state certain generalized variants of the Mourre estimate which contain estimates of the commutator $i\left[H_{a}, A\right]$ depending on the fibers. There will be actually 4 variants. Two of them estimate the commutator from below-this is the usual case. The other two estimate the commutator from above. They are generalizations of the so-called reverse Mourre estimate, which was first introduced in [SigSof1] where it was used in the proof of the propagation theorem for $N$-body Schrödinger operators.

Let us introduce certain functions that "measure" the positivity of $i\left[H_{a}, A\right]$. Let $c$ be a number and $a \in \mathscr{A}$. Introduce the functions $\zeta_{a}^{c}$ on $K_{a}$ such that if $a \neq a_{\max }$ then

$$
\zeta_{a}^{c}\left(k_{a}\right)= \begin{cases}k_{a} \cdot \nabla \eta_{k_{a}}\left(k_{a}\right) & \text { if } k_{a} \in E S_{a}^{p p}\left(E_{0}\right), \\ c & \text { otherwise }\end{cases}
$$

and

$$
\zeta_{a_{\max }}^{c}= \begin{cases}0 & \text { if } E_{0} \text { is an eigenvalue of } H \\ c & \text { otherwise. }\end{cases}
$$

Let $\kappa>0$. Put

$$
\begin{aligned}
& \tilde{\theta}_{a}^{c, \kappa}\left(k_{a}\right)=\inf \left\{\zeta_{b}^{c}\left(p_{b}\right): b \varsubsetneqq a, p_{b} \in K_{b},\left|\pi_{a} p_{b}-k_{a}\right|<\kappa\right\}, \\
& \theta_{a}^{c, \kappa}\left(k_{a}\right)=\inf \left\{\zeta_{b}^{c}\left(p_{b}\right): b \subset a, p_{b} \in K_{b},\left|\pi_{a} p_{b}-k_{a}\right|<\kappa\right\}, \\
& \tilde{\rho}_{a}^{c, \kappa}\left(k_{a}\right)=\sup \left\{\zeta_{b}^{c}\left(p_{b}\right): b \varsubsetneqq a, p_{b} \in K_{b},\left|\pi_{a} p_{b}-k_{a}\right|<\kappa\right\}
\end{aligned}
$$

and

$$
\rho_{a}^{c, \kappa}\left(k_{a}\right)=\sup \left\{\zeta_{b}^{c}\left(p_{b}\right): b \subset a, p_{b} \in K_{b},\left|\pi_{a} p_{b}-k_{a}\right|<\kappa\right\} .
$$

Now we can state our generalized variants of the Mourre estimate. 
Theorem 5.2. Assume A1, A2, A3, B1, and $\mathrm{C} 1$. Let $a \in \mathscr{A}, \varepsilon, \kappa>0$ and $c_{ \pm} \in \mathbf{R}$. Then the following statements are true:

(a) There exists an open interval $\Delta$ containing $E_{0}$ and a-compact operators $B_{a}^{ \pm}$such that

$$
\begin{aligned}
& E_{\Delta}\left(H_{a}\right)\left(\tilde{\rho}_{a}^{c_{+}, \kappa}\left(D_{a}\right)+\varepsilon\right)+E_{\Delta}\left(H_{a}\right) B_{a}^{+} E_{\Delta}\left(H_{a}\right) \\
& \quad \geq E_{\Delta}\left(H_{a}\right) i\left[H_{a}, A\right] E_{\Delta}\left(H_{a}\right) \\
& \quad \geq E_{\Delta}\left(H_{a}\right)\left(\tilde{\theta}^{c_{-}, \kappa}\left(D_{a}\right)-\varepsilon\right)+E_{\Delta}\left(H_{a}\right) B_{a}^{-} E_{\Delta}\left(H_{a}\right) .
\end{aligned}
$$

(b) There exists an open interval $\Delta^{\prime}$ containing $E_{0}$ such that

$$
\begin{gathered}
E_{\Delta^{\prime}}\left(H_{a}\right)\left(\rho^{c_{+}, \kappa}\left(D_{a}\right)+\varepsilon\right) \geq E_{\Delta^{\prime}}\left(H_{a}\right) i\left[H_{a}, A\right] E_{\Delta^{\prime}}\left(H_{a}\right) \\
\geq E_{\Delta^{\prime}}\left(H_{a}\right)\left(\theta^{c_{-}, \kappa}\left(D_{a}\right)-\varepsilon\right) .
\end{gathered}
$$

The reason why we stated Theorem 5.2 for an arbitrary $a \in \mathscr{A}$ is twofold. First, the parts of this theorem which estimate the commutator from below facilitate the inductive proof of Theorem 5.1. In fact, Theorem 5.2(a) for $a=a_{\max }$ implies Theorem 5.1. Secondly, we think that Theorem 5.2 may turn out to be useful as it stands, for instance in some kinds of propagation theorems (compare [De1]).

This section and the next one contain the proof of Theorem 5.2. We will only prove the parts of the theorem that estimate the commutator from below, since the proof of the other parts is analogous.

In this section we will assume that (a) is proven and we will show (b). The proof will be based on the fact that both sides of (5.1) and (5.2) are $a$-fibered with compact supports in $K_{a}$. Thus it suffices to look at the problem "locally". This idea is the motivation for the following lemma.

Lemma 5.3. Suppose that the assumptions of Theorem 5.2 are satisfied. Suppose also that for some open interval $\Delta$ containing $E_{0}$ and an a-compact operator $B_{a}$

$$
E_{\Delta}\left(H_{a}\right) i\left[H_{a}, A\right] E_{\Delta}\left(H_{a}\right) \geq\left(\tilde{\theta}_{a}^{c, \kappa}\left(D_{a}\right)-\varepsilon / 2\right) E_{\Delta}\left(H_{a}\right)+E_{\Delta}\left(H_{a}\right) B_{a} E_{\Delta}\left(H_{a}\right) .
$$

Then for any $k_{a} \in K_{a}$ there exists $\delta_{k_{a}}>0$ and an open interval $\Delta_{k_{a}}$ containing $E_{0}$ such that

$$
\begin{aligned}
& E_{B\left(k_{a}, \delta_{k_{a}}\right)}\left(D_{a}\right) E_{\Delta_{k_{a}}}\left(H_{a}\right) i\left[H_{a}, A\right] E_{\Delta_{k_{a}}}\left(H_{a}\right) \\
& \quad \geq E_{B\left(k_{a}, \delta_{k_{a}}\right)}\left(D_{a}\right) E_{\Delta_{k_{a}}}\left(H_{a}\right)\left(\theta_{a}^{c, k}\left(D_{a}\right)-\varepsilon\right) .
\end{aligned}
$$

Proof. Assume first that $k_{a} \in E S_{a}^{p p}\left(E_{0}\right)$. Choose $\delta_{0}$ such that $\kappa \geq \delta_{0}>0$ and $B\left(k_{a}, \delta_{0}\right) \subset U_{k_{a}}$. Lemma A1 guarantees that there exist norm-continuous functions

$$
B\left(k_{a}, \delta_{0}\right) \ni p_{a} \mapsto P^{N}\left(p_{a}\right) \in B\left(L^{2}\left(X^{a}\right)\right)
$$

with values in finite-dimensional projections such that $s-\lim _{N \rightarrow \infty} P^{N}\left(p_{a}\right)=$ $P_{k_{a}}\left(p_{a}\right)$ and if $N<M$ then $P^{N}\left(p_{a}\right) \leq P^{M}\left(p_{a}\right)$. 
Choose $f \in C_{0}\left(K_{a}\right)$ such that $\operatorname{supp} f \subset B\left(k_{a}, \delta_{0}\right)$ and $f\left(k_{a}\right)=1$. Define $F=\int_{K_{a}}^{\oplus} P_{k_{a}}\left(p_{a}\right) f\left(p_{a}\right) d p_{a}$ and $F^{N}=\int_{K_{a}}^{\oplus} P^{N}\left(p_{a}\right) f\left(p_{a}\right) d p_{a}$.

If $k_{a} \notin E S_{a}^{p p}\left(E_{0}\right)$ we just set $F=F^{N}=0$.

Note that $F$ is $a$-continuous and the $F^{N}$ 's are $a$-compact.

For shortness we will write $E_{\Delta}$ instead of $E_{\Delta}\left(H_{a}\right)$. Now write

$$
E_{\Delta} i\left[H_{a}, A\right] E_{\Delta}=C_{1}+C_{2}+C_{2}^{*}+C_{3}+C_{3}^{*}+C_{4}
$$

where

$$
\begin{aligned}
& C_{1}=E_{\Delta}\left(1-F^{N}\right) i\left[H_{a}, A\right]\left(1-F^{N}\right) E_{\Delta}, \\
& C_{2}=E_{\Delta}(1-F) i\left[H_{a}, A\right] F^{N} E_{\Delta}, \\
& C_{3}=E_{\Delta}\left(F-F^{N}\right) i\left[H_{a}, A\right] F^{N} E_{\Delta}, \\
& C_{4}=E_{\Delta} F^{N} i\left[H_{a}, A\right] F^{N} E_{\Delta} .
\end{aligned}
$$

We can apply Theorem 4.1 to the last three terms on the right-hand side of (5.4). Thus

$$
C_{3}+C_{3}^{*}+C_{4}=D_{a} \cdot \nabla \eta_{k_{a}}\left(D_{a}\right) E_{\Delta} F^{N} .
$$

By the continuity of $p_{a} \mapsto p_{a} \cdot \nabla \eta_{k_{a}}\left(p_{a}\right)$, if $\delta$ is small enough we can write

$$
E_{B\left(k_{a}, \delta\right)}\left(D_{a}\right)\left(C_{3}+C_{3}^{*}+C_{4}\right) \geq E_{B\left(k_{a}, \delta\right)}\left(D_{a}\right) E_{\Delta} F^{N}\left(k_{a} \cdot \nabla \eta_{k_{a}}\left(k_{a}\right)-\varepsilon / 2\right) .
$$

If $\delta<\kappa$ then the right-hand side of $(5.5)$ is greater or equal than

$$
E_{B\left(k_{a}, \delta\right)}\left(D_{a}\right) E_{\Delta} F^{N}\left(\theta_{a}^{c, \kappa}\left(D_{a}\right)-\varepsilon / 2\right) .
$$

This takes care of the last three terms on the right-hand side of (5.4). It remains to handle the first three terms. By (5.3) we can write

$$
C_{1} \geq E_{\Delta}\left(1-F^{N}\right)\left(\tilde{\theta}_{a}^{c, \kappa}\left(D_{a}\right)-\varepsilon / 2\right)+E_{\Delta}\left(1-F^{N}\right) B_{a}\left(1-F^{N}\right) E_{\Delta} .
$$

Thus the proof of our lemma will be completed if we show the following lemma, which is based on an idea due to B. Simon (see [FH1]).

Lemma 5.4. There exists $\delta>0, N \in \mathbb{N}$ and an open interval $\Delta_{k_{a}}$ containing $E_{0}$ such that

$$
\left\|E_{B\left(k_{a}, \delta\right)}\left(D_{a}\right) E_{\Delta_{k_{a}}}\left[\left(1-F^{N}\right) B_{a}\left(1-F^{N}\right)+C_{2}+C_{2}^{*}\right] E_{\Delta_{k_{a}}}\right\|<\varepsilon / 2 .
$$

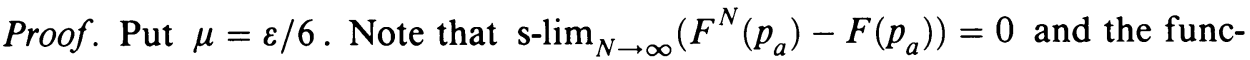
tion $p_{a} \mapsto F^{N}\left(p_{a}\right)-F\left(p_{a}\right)$ is norm-continuous. Thus by Lemma 2.2 we can find $\delta>0$ and $N \in \mathbb{N}$ such that

$$
\left\|E_{B\left(k_{a}, \delta\right)}\left(D_{a}\right)\left[\left(1-F^{N}\right) B_{a}\left(1-F^{N}\right)-(1-F) B_{a}(1-F)\right]\right\|<\mu .
$$

Next choose $g \in C_{0}(\mathbb{R})$ such that $g\left(H_{a}-E_{0}\right) E_{\Delta}=E_{\Delta}$. Define

$$
\tilde{B}=(1-F) g\left(H_{a}-E_{0}\right) i\left[H_{a}, A\right] F^{N} .
$$


Note that $\tilde{B}$ is $a$-compact. The inequality

$$
\left(\mu^{1 / 2} F^{N} \pm \mu^{-1 / 2} \tilde{B}\right)\left(\mu^{1 / 2} F^{N} \pm \mu^{-1 / 2} \tilde{B}^{*}\right) \geq 0
$$

implies

$$
\begin{aligned}
& \mu F^{N} E_{\Delta}+\mu^{-1} E_{\Delta}(1-F) \tilde{B} \tilde{B}^{*}(1-F) E_{\Delta} \\
& \quad \geq C_{2}+C_{2}^{*} \geq-\mu F^{N} E_{\Delta}-\mu^{-1} E_{\Delta}(1-F) \tilde{B} \tilde{B}^{*}(1-F) E_{\Delta} .
\end{aligned}
$$

It remains to show that we will find $\delta>0$ and an open interval $\Delta_{k_{a}}$ containing $E_{0}$ such that

$$
\left\|E_{B\left(k_{a}, \delta\right)}\left(D_{a}\right) E_{\Delta_{k_{a}}}(1-F)\left(B_{a} \pm \mu^{-1} \tilde{B} \tilde{B}^{*}\right)(1-F) E_{\Delta_{k_{a}}}\right\|<\mu .
$$

Clearly for any $\gamma>0$ the function $g\left(\left(H_{a}-E_{0}\right) / \gamma\right)(1-F)$ is $a$-continuous and

$$
\mathrm{s}-\lim _{\gamma \rightarrow 0} g\left(\frac{H_{a}-E_{0}}{\gamma}\right)\left(k_{a}\right)\left(1-F\left(k_{a}\right)\right)=0 .
$$

Moreover, the operators $B_{a} \pm \mu^{-1} \tilde{B} \tilde{B}^{*}$ are $a$-compact. Thus another application of Lemma 2.2 shows that we can choose $\delta>0$ and $\gamma>0$ small enough such that

$$
\left\|E_{B\left(k_{a}, \delta\right)}\left(D_{a}\right) g\left(\frac{H_{a}-E_{0}}{\gamma}\right)(1-F)\left(B-\mu^{-1} \tilde{B} \tilde{B}^{*}\right)(1-F) g\left(\frac{H_{a}-E_{0}}{\gamma}\right)\right\|<\mu .
$$

This implies (5.6) and ends the proof of Lemma 5.4. Q.E.D.

Proof of.Theorem 5.2(b) given Theorem 5.2(a). Let $\Delta_{0}$ be a certain open interval containing $E_{0}$. For any $k_{a} \in K_{a}$ let $\delta_{k_{a}}$ and $\Delta_{k_{a}}$ be determined in the obvious way by Lemma 5.3. We may assume that $\Delta_{k_{a}} \subset \Delta_{0}$.

The set $\overline{\bigcup_{E \in \Delta_{0}} E S_{a}(E)}$ is compact and the family $B\left(k_{a}, \delta_{k_{a}}\right)$ for $k_{a} \in K_{a}$ is its open cover. Choose a finite subcover labelled by $k_{a}^{1}, \ldots, k_{a}^{N}$. Now if we put $\Delta^{\prime}=\bigcap_{i=1}^{N} \Delta_{k_{a}^{i}}$ then by Lemma 5.3 inequality (5.2) is true. Q.E.D.

\section{GEOMETRIC METHODS}

In this section we complete the proof of Theorem 5.2. The proof will use the induction with respect to \#a. It will be based on the so-called geometric method, which has proved very fruitful in the study of $N$-body Schrödinger operators (see e.g. [E, DS, Sim, Sig2, PSS, FH1, CFKS]). Like in the previous section we will follow very closely the line of argument of [FH1].

An important tool that is used in the geometric method is an appropriate partition of unity in the configuration space. We will need to construct a whole family of partitions of unity indexed by $a \in \mathscr{A}$. This is the subject of the following lemma (which belongs to the folklore of the geometric method-see e.g. [FH1, PSS]). 
Lemma 6.1. Let $a \in \mathscr{A}$. There exists a family $\left\{\chi_{b}^{a} \in C^{\infty}\left(X^{a}\right): \# b=\# a+1\right.$, $b \subset a\}$ such that

(a) $\left|\partial^{a} \chi_{b}^{a}\right| \leq c_{\alpha}\left(\left|x^{a}\right|+1\right)^{-|\alpha|}$ for all $\alpha$,

(b) for any $b_{1} \subset a$ such that $b_{1} \not \subset b \subset a$ there exists $\varepsilon>0$ such that $\left|\pi^{b_{1}} x^{a}\right| \geq \varepsilon\left|x^{a}\right|-1$ on $\operatorname{supp} \chi_{b}^{a}$.

Proof. Let $S^{a}$ denote the unit sphere in $X^{a}$. Then $X_{b}^{a} \cap S^{a}$ with $\# b=\# a+1$ and $b \subset a$ are closed and disjoint for distinct $b$ 's. Let $\tilde{\chi}_{b}^{a} \in C^{\infty}\left(S^{a}\right)$ have the property that

$$
\sum_{\substack{\sharp b=\# a+1 \\ b \subset a}}\left(\tilde{\chi}_{b}^{a}\right)^{2}=1
$$

and $\tilde{\chi}_{b}^{a}=1$ on a neighborhood of $X_{b}^{a} \cap S^{a}$. Now it is enough to take $\chi_{b}^{a} \in$ $C^{\infty}\left(X^{a}\right)$ such that $\chi_{b}^{a}\left(x^{a}\right)=\tilde{\chi}_{b}^{a}\left(x^{a} /\left|x^{a}\right|\right)$ for $\left|x^{a}\right| \geq 1$. Q.E.D.

The following lemma is quite easy in the nondispersive case. In the dispersive case it follows from Appendix 2 by the methods of pseudodifferential operators.

Lemma 6.2. (a) Assume $\mathrm{A} 1$ and $\mathrm{A} 2$. Then $\left[H_{0}, \chi_{b}^{a}\left(x^{a}\right)\right]\left(H_{0}+1\right)^{-1}$ is a-compact.

(b) Assume A2 and A3. Then $\left[\left[H_{0}, A\right], \chi_{b}^{a}\left(x^{a}\right)\right]\left(H_{0}+1\right)^{-1}$ is a-compact.

The next lemma is an analog of Lemma 2.2 of [FH1]. We omit its proof, which given Lemma 6.2 is very similar to that contained in [FH1].

Lemma 6.3. Let $b, b_{1} \subset a$ and $F \in C_{0}^{\infty}(\mathbb{R})$. Assume $\mathrm{A} 1$ and $\mathrm{A} 2$. Then

(a) $\left[F\left(H_{b}\right), \chi_{b_{1}}^{a}\left(\pi^{a} x\right)\right]\left(H_{0}+1\right)$ is a-compact, and

(b) $\chi_{b}^{a}\left(\pi^{a} x\right)\left[F\left(H_{a}\right)-F\left(H_{b}\right)\right]\left(H_{0}+1\right)$ is a-compact.

(c) If moreover $\mathrm{B} 1$ is true then $F\left(H_{a}\right) \chi_{b}^{a}\left(\pi^{a} x\right) \pi^{a} x \cdot \nabla I_{b}^{a}\left(\pi^{a} x\right) \chi_{b}^{a}\left(\pi^{a} x\right) F\left(H_{a}\right)$ is a-compact.

An analog of the following lemma is also contained in [FH1].

Lemma 6.4. Let $F \in C_{0}^{\infty}(\mathbb{R})$ and $a \in \mathscr{A}$. Assume $\mathrm{A} 1, \mathrm{~A} 2$ and $\mathrm{B} 1$. Then there exist a-compact operators $B_{a}$ and $\tilde{B}_{a}$ such that

(a)

$$
F\left(H_{a}\right) i\left[H_{a}, A\right] F\left(H_{a}\right)=\sum_{\substack{b \subset a \\ \# b=\# a+1}} \chi_{b}^{a}\left(\pi^{a} x\right) F\left(H_{b}\right) i\left[H_{b}, A\right] F\left(H_{b}\right) \chi_{b}^{a}\left(\pi^{a} x\right)+B_{a}
$$

and

(b)

$$
F^{2}\left(H_{a}\right)=\sum_{\substack{b \subset a \\ \# b=\# a+1}} \chi_{b}^{a}\left(\pi^{a} x\right) F^{2}\left(H_{b}\right) \chi_{b}^{a}\left(\pi^{a} x\right)+\tilde{B}_{a} .
$$

Proof. We can write

$$
\left[H_{a}, A\right]=\sum_{\substack{b \subset a \\ \# b=\# a+1}} \chi_{b}^{a}\left[H_{a}, A\right] \chi_{b}^{a}+C
$$


where

$$
C=\sum_{\substack{b \subset a \\ \sharp b=\# a+1}} \chi_{b}^{a}\left[\left[H_{0}, A\right], \chi_{b}^{a}\right] .
$$

(Note that (6.1) is a generalization of the so-called IMS localization formulasee e.g. [Sig2, CFKS].) By Lemma 6.2(b) $C\left(H_{0}+1\right)^{-1}$ is $a$-compact. Thus

$$
F\left(H_{a}\right) i\left[H_{a}, A\right] F\left(H_{a}\right)=\sum_{\substack{b \subset a \\ \# b=\# a+1}} F\left(H_{a}\right) \chi_{b}^{a} i\left[H_{a}, A\right] \chi_{b}^{a} F\left(H_{a}\right)+C_{1}
$$

where $C_{1}$ is $a$-compact. Now (a) follows from (6.2) by Lemma 6.3. Part (b) has a similar proof. Q.E.D.

Now we are ready for the proof of Theorem 5.2.

Proof of Theorem 5.2. We use induction with respect to \#a. We start with the maximal \#a, that is, we consider first $a=a_{\min }$. In this case $\tilde{\theta}_{a_{\min }}^{c, \kappa}=c$. Choose an arbitrary bounded interval $\Delta$ containing $E_{0}$. Then

$$
E_{\Delta}\left(H_{0}\right) i\left[H_{0}, A\right] E_{\Delta}\left(H_{0}\right)-\left(\tilde{\theta}_{a_{\min }}^{c, \kappa}-\varepsilon\right) E_{\Delta}\left(H_{0}\right)=g(D)
$$

where $g$ is a bounded function on $K$ with a compact support. Let us choose a continuous function $f$ on $K$ such that $\lim _{|k| \rightarrow \infty} f(k)=0$ and $f \leq g$. Set $B_{a_{\min }}=f(D)$. Then $B_{a_{\min }}$ is $a_{\min }$-compact and (5.1) is satisfied. Thus we proved (a) in the case $a=a_{\min }$.

Now assume that $a$ is a certain fixed element of $\mathscr{A}$ and (b) is true for all $b \subseteq a$ such that $\# b=\# a+1$. In other words, there exists an open interval $\Delta_{1}$ containing $E_{0}$ such that if $b \subseteq a$ and $\# b=\# a+1$ then

$$
E_{\Delta_{1}}\left(H_{b}\right) i\left[H_{b}, A\right] E_{\Delta_{1}}\left(H_{b}\right) \geq\left(\theta^{c, \kappa / 2}\left(D_{b}\right)-\varepsilon\right) E_{\Delta_{1}}\left(H_{b}\right)
$$

Let $\Delta_{2}$ be an open interval containing $E_{0}$ such that $\bar{\Delta}_{2} \subset \Delta_{1}$. Let $F \subset$ $C_{0}^{\infty}(\mathbf{R})$ have the property that $\operatorname{supp} F \subset \Delta_{1}$ and $F=1$ on $\Delta_{2}$.

Clearly $\theta_{b}^{c, \kappa / 2} \geq \tilde{\theta}_{a}^{c, \kappa / 2}$ for $b \subset a$ such that $\# b=\# a+1$. By Lemma A3.1 we can choose a bounded continuous function $\xi$ on $K_{a}$ such that

$$
\tilde{\theta}_{a}^{c, \kappa / 2}-\varepsilon \geq \xi \geq \tilde{\theta}_{a}^{c, \kappa}-\varepsilon .
$$

Now we can write

$$
\sum_{\substack{b \subset a \\ \# b=\# a+1}} \chi_{b}^{a} F\left(H_{b}\right) i\left[H_{b}, A\right] F\left(H_{b}\right) \chi_{b}^{a} \geq \sum_{\substack{b \subset a \\ \# b=\# a+1}} \chi_{b}^{a} F\left(H_{b}\right) \xi\left(D_{a}\right) F\left(H_{b}\right) \chi_{b}^{a} .
$$

By Lemma 6.4(a) the left-hand side of (6.3) is equal to

$$
F\left(H_{a}\right) i\left[H_{a}, A\right] F\left(H_{a}\right)-B_{a}
$$

and the right-hand side of (6.3) is equal to

$$
F^{2}\left(H_{a}\right) \xi\left(D_{a}\right)-\tilde{B}_{a} \xi\left(D_{a}\right),
$$


where $B_{a}$ and $\tilde{B}_{a}$ are $a$-compact. Since $\xi\left(D_{a}\right)$ is $a$-continuous, $\tilde{B}_{a} \xi\left(D_{a}\right)$ is $a$-compact. Clearly (6.5) is greater or equal than

This implies (5.2).

$$
F^{2}\left(H_{a}\right)\left(\tilde{\theta}_{a}^{c, \kappa}\left(D_{a}\right)-\varepsilon\right)-\tilde{B}_{a} \xi\left(D_{a}\right) .
$$

Thus we have shown that Theorem $5.2(\mathrm{a})$ is true for this $a \in \mathscr{A}$. In $\S 5$ we proved that Theorem 5.2(a) implies (b). Thus the proof of our theorem is complete. Q.E.D.

\section{Consequences of Mourre estimate}

The Mourre estimate itself may not look like a very interesting fact. Starting from the Mourre estimate though we can prove a number of deep results about the spectral properties of the operator $H$. In the context of the nondispersive $N$-body Schrödinger operators those properties were first proved by E. Mourre in [M1, M2] (see also [PSS, CFKS, JP, JMP, SigSof2]). Often they can be formulated as quite abstract theorems with $H$ and $A$ being selfadjoint operators satisfying rather general assumptions including the Mourre estimate on $\Delta \subset \mathbb{R}$. In this section we apply those theorems to dispersive $N$-body Schrödinger operators. We obtain a number of interesting theorems about those operators, which are the main results of our paper.

The first result follows from the Mourre estimate and the virial theorem by an argument due to E. Mourre [M1, PSS, CFKS]. For the convenience of the reader we repeat this argument in our paper.

Theorem 7.1. Assume A1, A2, A3, B1, C1, and $\mathrm{C} 2$. Then there exists an open interval $\Delta$ containing $E_{0}$ such that $H$ has at most finitely many eigenvalues in $\Delta$ and each eigenvalue has a finite multiplicity.

Proof. By Theorem 5.1 there exists an open interval $\Delta$ containing $E_{0}$ such that $H$ satisfies the Mourre estimate on $\Delta$. Let $\left\{\psi_{n}\right\}$ be an infinite family of orthonormal eigenfunctions of $H$ with eigenvalues in $\Delta$. Then by Theorem 4.1b) and the Mourre estimate

$$
0=\left(\psi_{n}, i[H, A] \psi_{n}\right) \geq c\left\|\psi_{n}\right\|^{2}+\left(\psi_{n}, B \psi_{n}\right) .
$$

Since $\psi_{n} \rightarrow 0$ weakly and $B$ is compact, the second term of $(7.1)$ goes to zero. This is impossible because $c\left\|\psi_{n}\right\|^{2}=c>0$. Q.E.D.

Another application of the Mourre estimate is also due to E. Mourre [M1, M2]. Later on it was improved in [PSS, Ya, JP, JPM] (see also [CFKS]).

Theorem 7.2. Assume $\mathrm{A} 1, \mathrm{~A} 2, \mathrm{~A} 3, \mathrm{~A} 4, \mathrm{~B} 1, \mathrm{~B} 1^{\prime}, \mathrm{B} 2, \mathrm{C} 1$, and $\mathrm{C} 2$. Assume also that $E_{0}$ is not an eigenvalue of $H$ and $\varepsilon>0$. Then there exists an open interval $\Delta$ containing $E_{0}$ such that

$$
\varlimsup_{\delta \downarrow 0} \sup _{\mu \in \Delta}\left\|(|A|+1)^{-1 / 2-\varepsilon}(H-\mu-i \delta)^{-1}(|A|+1)^{-1 / 2-\varepsilon}\right\|<\infty .
$$

The above theorem is a consequence of the following theorem proved in [PSS] (see also [M1, M2, CFKS]). 
Theorem 7.3. Suppose that $H$ and $A$ are selfadjoint operators on $\mathscr{H}, \Delta$ and $\Delta_{1}$ are open intervals in $\mathbb{R}$ such that $\bar{\Delta} \subset \Delta_{1}$ and $\varepsilon>0$. Let $\mathscr{H}_{m}$ be the scale of spaces associated with the operator $H$, namely

$$
\mathscr{H}_{m}=\left\{\psi \in \mathscr{H}:\left\|\left(H^{2}+1\right)^{m / 2} \psi\right\|<\infty\right\}
$$

for $m \geq 0$ and $\mathscr{H}_{-m}$ is the completion of $\mathscr{H}$ in the norm $\left\|\left(H^{2}+1\right)^{-m / 2} \psi\right\|$. Assume moreover the following conditions:

(1) $\mathscr{D}(A) \cap \mathscr{H}_{+2}$ is dense in $\mathscr{H}_{+2}$.

(2) The form $i[H, A]$ defined on $\mathscr{D}(A) \cap \mathscr{H}_{+2}$ extends to a bounded operator from $\mathscr{H}_{+2}$ to $\mathscr{H}_{-1}$.

(3) There is a selfadjoint operator $H_{0}$ with $\mathscr{D}\left(H_{0}\right)=\mathscr{D}(H)$ such that $i\left[H_{0}, A\right]$ extends to a bounded map from $\mathscr{H}_{+2}$ to $\mathscr{H}$ and $\mathscr{D}(A) \cap \mathscr{D}\left(H_{0} A\right)$ is a core for $H_{0}$.

(4) The form $[[H, A], A]$ where $[H, A]$ is as in (2) extends from $\mathscr{H}_{+2} \cap \mathscr{D}(A)$ to a bounded map from $\mathscr{H}_{+2}$ to $\mathscr{H}_{-2}$.

(5) There exists a positive number $c$ such that

$$
E_{\Delta_{1}}(H) i[H, A] E_{\Delta_{1}}(H) \geq c E_{\Delta_{1}}(H) .
$$

Then

$$
\varlimsup_{\delta \downarrow 0} \sup _{\mu \in \Delta}\left\|(|A|+1)^{-1 / 2-\varepsilon}(H-\mu-i \delta)^{-1}(|A|+1)^{-1 / 2-\varepsilon}\right\|<\infty .
$$

Proof of Theorem 7.2 given Theorem 7.3. Theorem 5.2(b) in the case $a=a_{\max }$ (which actually in this case can be viewed as a simple consequence of Theorem 5.1) guarantees the existence of an open interval $\Delta_{1}$ containing $E_{0}$ such that (7.2) is satisfied. Let $\Delta$ be an open interval such that $E_{0} \in \Delta$ and $\bar{\Delta} \subset \Delta_{1}$. We shall verify that all the assumptions of Theorem 7.3 are satisfied for our choice of $H, H_{0}, A, \Delta$ and $\Delta_{1}$.

First note that $\mathscr{D}\left(H_{0}\right)=\mathscr{D}(H)$. Thus by interpolation, if $|m| \leq 2$ then the norms $\left\|\left(H^{2}+1\right)^{m / 2} \psi\right\|$ and $\left\|\left(H_{0}^{2}+1\right)^{m / 2} \psi\right\|$ are equivalent. Now we see that (1) is true because $\mathscr{S}(X) \subset \mathscr{D}(A) \cap \mathscr{H}_{+2}$ and $\mathscr{S}(X)$ is dense in $\mathscr{H}_{+2}$. (2) follows from $\mathrm{A} 3$ and $\mathrm{B1}^{\prime}$. (3) follows from $\mathrm{A} 3$ and finally (4) follows from A4 and B2. Q.E.D.

Following [M1, PSS, CFKS] we state a number of straightforward consequences of Theorem 7.2.

Corollary 7.4. Suppose that the assumptions of Theorem 7.2 are true. Let $\Delta$ be the interval obtained in this theorem. Then

(a) there is no singular continuous spectrum in $\Delta$.

Assume moreover A5. Then

(b) $\varlimsup_{\delta \downarrow 0} \sup _{\mu \in \Delta}\left\|(|x|+1)^{-1 / 2-\varepsilon}(H-\mu-i \delta)^{-1}(|x|+1)^{-1 / 2-\varepsilon}\right\|<\infty$ and

(c) there exists $c$ such that if $\psi \in L^{2}(X)$ then

$$
\int_{-\infty}^{\infty}\left\|(|x|+1)^{-1 / 2-\varepsilon} e^{i H t} E_{\Delta}(H) \psi\right\|^{2} d t \leq c\|\psi\|^{2} .
$$


(This property is called the $H$-smoothness of $(|x|+1)^{-1 / 2-\varepsilon}$ on $\Delta$; see $[\mathrm{Ka} 1$, $\mathrm{Ka} 2$, RS4].)

Proof. (a) follows by the general criterion contained e.g. in [RS4, §XIIIb]. The proof of (b) mimicks the analogous proof from [PSS]. It is based on the following lemma.

Lemma 7.5. If $0 \leq \beta \leq 1$ then

$$
(|A|+1)^{\beta}(H+i)^{-1}(|x|+1)^{-\beta}
$$

is bounded.

Proof. Note that

$$
\begin{aligned}
D \cdot x(H+i)^{-1}(|x|+1)^{-1}= & D(H+i)^{-1} \cdot x(|x|+1)^{-1}+i D(H+i)^{-1} \\
& \cdot \nabla \omega(D)(H+i)^{-1}(|x|+1)^{-1} .
\end{aligned}
$$

Hypothesis A5 implies the boundedness of $D(H+i)^{-1}$. Thus (7.4) is bounded. This implies the boundedness of (7.3) for $\beta=1$. Now we apply the interpolation. Q.E.D.

Now Corollary 7.4(b) follows from Theorem 7.2 and Lemma 7.5 by an argument contained in the proof of Theorem 8.1 of [PSS]. Finally, (c) follows from (b) by the theory of Kato-smooth operators (see [Ka1, Ka2, RS4]). Q.E.D.

\section{APPENDIX 1}

In this appendix we prove a simple lemma on approximating projectionvalued functions by functions with values in finite-dimensional projections. Facts of this kind are well known e.g. in the $K$-theory of $C^{*}$-algebras.

Lemma A1.1. Let $U$ be a star-shaped compact subset of $\mathbb{R}^{n}$. Let $U \ni p \mapsto$ $P(p) \in B(\mathscr{H})$ be a norm-continuous function with values in orthogonal projections. Then there exists a sequence of norm-continuous functions $U \ni p \mapsto$ $P^{N}(p) \in B(\mathscr{H})$ with values in finite-dimensional projections such that if $N \leq M$ then $P^{N}(p) \leq P^{M}(p)$ and $s-\lim _{N \rightarrow \infty} P^{N}(p)=P(p)$.

Proof. It is enough to assume that $U=B(0,1)$. Let $p, p^{\prime} \in B(0,1)$ and $\left\|P(p)-P\left(p^{\prime}\right)\right\|<1$. Then

$$
\left\|1-P\left(p^{\prime}\right) P(p) P\left(p^{\prime}\right)-\left(1-P\left(p^{\prime}\right)\right)(1-P(p))\left(1-P\left(p^{\prime}\right)\right)\right\|<1 .
$$

Thus we can define

$$
A\left(p^{\prime}, p\right)=\left[P\left(p^{\prime}\right) P(p) P\left(p^{\prime}\right)+\left(1-P\left(p^{\prime}\right)\right)(1-P(p))\left(1-P\left(p^{\prime}\right)\right)\right]^{-1 / 2} P\left(p^{\prime}\right) P(p) .
$$

Note that $A\left(p^{\prime}, p\right)$ is a partial isometry from $\operatorname{Ran} P(p)$ to $\operatorname{Ran} P\left(p^{\prime}\right), A(p, p)$ $=P(p)$ and $A\left(p^{\prime}, p\right)$ depends norm-continuously on both parameters as long as $\left\|P(p)-P\left(p^{\prime}\right)\right\|<1$. 
By the compactness of $B(0,1)$ we can choose $n \in \mathbb{N}$ such that if $p, p^{\prime} \in$ $B(0,1)$ and $\left|p-p^{\prime}\right|<\frac{1}{n}$ then $\left\|P(p)-P\left(p^{\prime}\right)\right\|<1$. Now for $p \in B(0,1)$ we can define

$$
R(p)=A\left(p, \frac{k}{n} \frac{p}{|p|}\right) A\left(\frac{k}{n} \frac{p}{|p|}, \frac{(k-1)}{n} \frac{p}{|p|}\right) \cdots A\left(\frac{1}{n} \frac{p}{|p|}, 0\right)
$$

where $k$ is the biggest integer less than $n|p|$. Clearly, $R(p)$ depends normcontinuously on $p, R^{*}(p) R(p)=P(0)$ and $R(p) R^{*}(p)=P(p)$.

Now let $P^{N}$ be an increasing sequence of finite-dimensional projections such that $\mathrm{s}-\lim _{N \rightarrow \infty} P^{N}=P(0)$. Then we can put $P^{N}(p)=R(p) P^{N} R^{*}(p)$. Q.E.D.

\section{APPENDIX 2}

This appendix contains the elements of the theory of pseudodifferential operators that we need to prove Lemma 6.2. Our approach is based on [Hö] (see also [Ta]).

Unfortunately, the results that we need in our paper do not follow easily from those contained in [Hö], although they are very similar to them. This is why we essentially have to begin at the very foundations of the theory of pseudodifferential equations.

We start with introducing a certain family of generalized Sobolev spaces. Let $Z$ be a subspace of a certain finite-dimensional vector space $Y$ and $\xi$ a positive continuous function on $Y$. Let

$$
\left\{e_{i}: i=1, \ldots, n\right\}
$$

be a basis of $Z$. Define

$$
\mathscr{L}(Y, Z, \xi)=\left\{u \in L_{\mathrm{loc}}^{\infty}(Y): \text { if } m=0,1, \ldots \text { then }\|u\|_{\xi, m}<\infty\right\}
$$

where

$$
\|u\|_{\xi, m}=\sum_{k=0}^{m} \sum_{i_{1}, \ldots, i_{k}=1}^{n}\left\|\frac{\left(e_{i_{1}} \cdot \nabla\right) \cdots\left(e_{i_{k}} \cdot \nabla\right) u}{\xi}\right\|_{\infty} .
$$

Define also

$$
\begin{aligned}
& \mathscr{L}_{\infty}(Y, Z, \xi)=\left\{u \in C(Y): \text { if } i_{1}, \ldots, i_{k}=1, \ldots, n\right. \\
& \left.\operatorname{then} \frac{\left(e_{i_{1}} \cdot \nabla\right) \cdots\left(e_{i_{k}} \cdot \nabla\right) u(y)}{\xi(y)} \underset{|y| \rightarrow \infty}{\longrightarrow} 0\right\} .
\end{aligned}
$$

Note that $\mathscr{L}(Y, Z, \xi)$ is a Fréchet space and $\mathscr{L}_{\infty}(Y, Z, \xi)$ is its closed subspace. We will write $\mathscr{L}(Y, \xi)$ and $\mathscr{L}_{\infty}(Y, \xi)$ instead of $\mathscr{L}(Y, Y, \xi)$ and $\mathscr{L}_{\infty}(Y, Y, \xi)$.

We say that a positive continuous function $\xi$ on $Y$ is $Z$-slowly varying if and only if there exist $C$ and $N$ such that for any $y \in Y$ and $z \in Z$

$$
\xi(y) \leq C(1+|z|)^{N} \xi(y+z) .
$$


Instead of saying that $\xi$ is $Y$-slowly varying on $Y$ we will just say that $\xi$ is slowly varying.

For further reference we need the following lemma.

Lemma A2.1. Let $\xi$ be a $Z$-slowly varying function on $Y$. Then there exists $\tilde{\xi} \in \mathscr{L}(Y, Z, \xi)$ such that $\xi \leq \tilde{\xi} \leq c_{1} \xi$.

Proof. Let $j \in C_{0}^{\infty}(Z)$ have the property that $j \geq 0$ and $\int j(z) d z>0$. Then we can set

$$
\tilde{\xi}(y)=\left(\int_{Z} d z C^{-1}(1+|z|)^{-N} j(z)\right)^{-1} \int_{Z} d z \xi(y-z) j(z) \text {. Q.E.D. }
$$

Now let $B$ be a quadratic form on the space dual to $Y$. We define $e^{i B(D)}$ to be the map

$$
\mathscr{S}^{\prime}(Y) \ni \varphi \mapsto \mathscr{F}^{-1} e^{i B(\cdot)} \mathscr{F} \varphi \in \mathscr{S}^{\prime}(Y)
$$

where $\mathscr{F}$ denotes the Fourier transformation and $\mathscr{S}^{\prime}(Y)$ is the space of tempered distributions on $Y$. Clearly, $e^{i B(D)}$ is continuous on $\mathscr{S}^{\prime}(Y)$. Following [Hö] we will call $e^{i B(D)} \varphi$ the Gauss transform of $\varphi$.

Now we state a theorem about the Gauss transform on the spaces $\mathscr{L}(Y, Z, \xi)$ that is very useful in the study of the pseudodifferential operators.

Theorem A2.2. Let $\xi$ be a $Z$-slowly varying function on $Y$. Let $B$ be a quadratic form on the space dual to $Y$ such that if $k$ belongs to the annihilator of $Z$ then $B(k)=0$. Then $e^{i B(D)}$ is a continuous map of $\mathscr{L}(Y, Z, \xi)$ into itself and $\mathscr{L}_{\infty}(Y, Z, \xi)$ is its invariant subspace.

The above theorem is very closely related to the results contained in $\S 18.4$ of [Hö].

The proof of Theorem A2.2 is based on the fact that we can identify the form $B$ with a unique form acting on the space dual to $Z$. This enables us to treat $e^{i B(D)}$ as a direct integral of operators acting on fibers isomorphic to $\mathscr{L}(Z, \xi)$.

First let us show the following easy lemma based on the Sobolev estimate and the unitarity of $e^{i B(D)}$.

Lemma A2.3. Suppose that $l$ is a nonnegative integer, $R \in \mathbb{R}, k>\frac{n}{2}+l$, $u \in C^{k}(Z)$ and supp $u \subset B(0, R)$. Then

$$
\left\|(1+|z|)^{l} e^{i B(D)} u\right\|_{\infty} \leq c \sum_{|\alpha| \leq k}\left\|\partial^{\alpha} u\right\|_{\infty} .
$$

Proof. First note that

$$
\left\|(1+|z|)^{l} e^{i B(D)} u\right\|_{\infty} \leq c\left\|e^{i B(D)} u\right\|_{\infty}+c \sum_{i=1}^{n}\left\|\left(z_{i}\right)^{l} e^{i B(D)} u\right\|_{\infty} .
$$

By the Sobolev estimate if $k_{1}>\frac{n}{2}$ then

$$
\left\|e^{i B(D)} u\right\|_{\infty} \leq c \sum_{|\alpha| \leq k_{1}}\left\|e^{i B(D)} \partial_{z}^{\alpha} u\right\|_{2}
$$


and

$$
\left\|\left(z_{i}\right)^{l} e^{i B(D)} u\right\|_{\infty} \leq c \sum_{|\alpha| \leq k_{1}} \sum_{j=0}^{l}\left\|\left(z_{i}\right)^{j} e^{i B(D)} \partial_{z}^{\alpha} u\right\|_{2} .
$$

Next note that $\left[z_{i}, B(D)\right]$ is a first-order differential operator. This fact and the unitarity of $e^{i B(D)}$ imply

$$
\left\|\left(z_{i}\right)^{j} e^{i B(D)} \partial_{z}^{\alpha} u\right\|_{2} \leq \sum_{|\beta| \leq j} \sum_{j^{\prime}=0}^{j}\left\|\left(z_{i}\right)^{j^{\prime}} \partial_{z}^{\beta+\alpha} u\right\|_{2} .
$$

Finally, supp $u \subset B(0, R)$ implies

$$
\left\|\left(z_{i}\right)^{j^{\prime}} \partial_{z}^{\alpha+\beta} u\right\|_{2} \leq c_{j^{\prime}}\left\|\partial_{z}^{\alpha+\beta} u\right\|_{\infty} .
$$

If we put together (A2.3)-(A2.7) we obtain (A2.2). Q.E.D.

Our next lemma says roughly that if $\eta$ is slowly varying on $Z$ then $e^{i B(D)}$ is bounded on $\mathscr{L}(Z, \eta)$ and this boundedness is uniform in $\eta$ in a certain sense.

Lemma A2.4. Let

$$
M>\frac{3}{2} n+1+N
$$

and let $\eta$ be a positive continuous function on $Z$ such that for any $z, z^{\prime} \in Z$

$$
\eta(z) \leq C\left(1+\left|z-z^{\prime}\right|\right)^{N} \eta\left(z^{\prime}\right) .
$$

Then there exist $c_{j}$ such that if $j=0,1, \ldots$ and $u \in \mathscr{L}(Z, \eta)$ then

$$
\left\|e^{i B(D)} u\right\|_{\eta, j} \leq c_{j}\|u\|_{\eta, j+M} .
$$

Moreover, $c_{j}$ can be chosen to depend only on $j, N, n$ and $C$.

Proof. Let us identify $\mathbb{Z}^{n}$ with the subset of $Z$ consisting of the vectors with integral coordinates in the basis (A2.1). Let $r>0$ have the property that

$$
\bigcup_{\vec{k} \in \mathbf{Z}^{n}} B(\vec{k}, r)=Z \text {. }
$$

We can choose a partition of unity $j_{\vec{k}} \in C_{0}^{\infty}(Z)$ such that $\operatorname{supp} j_{\vec{k}} \subset B(\vec{k}, r)$, $\sum_{\vec{k} \in \mathbf{Z}^{n}} j_{\vec{k}}=1$ and $\left|\partial_{z}^{\alpha} j_{\vec{k}}\right| \leq c_{\alpha}$. Denote $j_{\vec{k}} u$ by $u_{\vec{k}}$. Then

$$
\begin{aligned}
\left\|\partial_{z}^{\alpha} u_{\vec{k}}\right\|_{\infty} & \leq c \sum_{|\beta| \leq|\alpha|} \sup _{z \in B(\vec{k}, r)}\left|\partial_{z}^{\beta} u(z)\right| \\
& \leq c^{\prime} \sup _{z \in B(\vec{k}, r)}|\eta(z)|\|u\|_{\eta,|\alpha|} \\
& \leq c^{\prime \prime} \eta(\vec{k})\|u\|_{\eta,|\alpha|} .
\end{aligned}
$$


Using Lemma A2.3, (A2.9) and (A2.11) we obtain

$$
\begin{aligned}
\left|\left(e^{i B(D)} u_{\vec{k}}\right)(z)\right| & \leq c(1+|z-\vec{k}|)^{-n-1-N} \sum_{|\alpha| \leq M}\left\|\partial_{z}^{\alpha} u_{\vec{k}}\right\|_{\infty} \\
& \leq c^{\prime}(1+|z-\vec{k}|)^{-n-1-N} \eta(\vec{k})\|u\|_{\eta, M} \\
& \leq c^{\prime \prime}(1+|z-\vec{k}|)^{-n-1} \eta(z)\|u\|_{\eta, M} .
\end{aligned}
$$

Thus for any $z$

$$
\sum_{\vec{k} \in \mathbf{Z}^{n}}\left(e^{i B(D)} u_{\vec{k}}\right)(z)
$$

is an absolutely convergent series. Set $\tilde{u}(z)$ to be equal to (A2.13). The estimate (A2.12) implies $\|\tilde{u}\|_{\eta, 0} \leq c\|u\|_{\eta, M}$. Clearly

$$
\lim _{R \rightarrow \infty} \sum_{|\vec{k}| \leq R} u_{\vec{k}}=u
$$

in $\mathscr{S}^{\prime}(Z)$. Thus

$$
\lim _{R \rightarrow \infty} \sum_{|\vec{k}| \leq R} e^{i B(D)} u_{\vec{k}}=e^{i B(D)} u .
$$

But we have just proved that the left-hand side of (A2.13) converges pointwise to a function that we called $\tilde{u}$. This shows that

$$
\left\|e^{i B(D)} u\right\|_{\eta, 0} \leq c\|u\|_{\eta, M} .
$$

If we replace $u$ with $\partial_{z}^{\alpha} u$ in the above estimate we obtain (A2.10). Q.E.D.

Now we are ready for the proof of Theorem A2.2.

Proof of Theorem A2.2. Clearly, we can represent $\mathscr{L}(Y, Z, \xi)$ as a direct integral with the fibers isomorphic to $\mathscr{L}\left(Z,\left.\xi\right|_{y+Z}\right)$. Moreover, $e^{i B(D)}$ acts separately in those fibers. Note that the restriction of $\xi$ to $y+Z$ for $y \in Y$ satisfies (A2.9) with the constants $C$ and $N$ that do not depend on $y \in Y$. Thus the boundedness of $e^{i B(D)}$ on $\mathscr{L}(Y, Z, \xi)$ follows immediately from Lemma A2.4.

To show that $\mathscr{L}_{\infty}(Y, Z, \xi)$ is invariant with respect to $e^{i B(D)}$ it suffices to note that it is the closure of $\mathscr{S}(Y)$ in $\mathscr{L}(Y, Z, \xi)$ and $e^{i B(D)}$ leaves $\mathscr{S}(Y)$ invariant. $(\mathscr{S}(Y)$ denotes the space of Schwartz test functions on $Y$.) Q.E.D.

Now we turn out attention to the main subject of this appendix, namely to the pseudodifferential operators. Let $X$ be a finite-dimensional vector space and $K$ its dual. Let $u \in \mathscr{S}^{\prime}(X \times K)$. We define $u(x, D)$ and $u(D, x)$ as the quadratic forms on $\mathscr{S}(X)$ such that if $\varphi, \psi \in \mathscr{S}(X)$ then

$$
(\varphi, u(x, D) \psi)=\frac{1}{(2 \pi)^{\operatorname{dim} X}} u\left(e^{i x k} \varphi \otimes \mathscr{F}(\psi)\right)
$$

and

$$
(\varphi, u(D, x) \psi)=u\left(e^{-i x k} \psi \otimes \mathscr{F}^{-1}(\varphi)\right) .
$$


Clearly, if $u$ is a sufficiently nice function then

$$
(\varphi, u(x, D) \psi)=\frac{1}{(2 \pi)^{\operatorname{dim} X}} \int \varphi(x) u(x, k) \psi(y) e^{i(x-y) k} d x d k d y
$$

and

$$
(\varphi, u(D, x) \psi)=\frac{1}{(2 \pi)^{\operatorname{dim} X}} \int \varphi(x) u(y, k) \psi(y) e^{i(x-y) k} d x d k d y .
$$

By a straightforward calculation one can show the following fact (see e.g. Theorem 18.1.7 of [Hö]).

Proposition A2.5. Let $u \in \mathscr{S}^{\prime}(X \times K)$ and $v=e^{i D_{x} \cdot D_{k}} u$. Then $v(x, D)=$ $u(D, x)$. Moreover $u(x, D)-u(D, x)=w(x, D)$ where $w=\int_{0}^{1} d \tau e^{i \tau D_{x} \cdot D_{k}} i D_{x}$. $D_{k} u$.

The following fact is a consequence of the Calderon-Vaillancourt theorem (see [Hö, Ta]) and of the compactness of operators with Hilbert-Schmidt kernels (see e.g. [Ka3]).

Theorem A2.6. (a) If $u \in \mathscr{L}(X \times K, 1)$ then both $u(x, D)$ and $u(D, x)$ extend to bounded operators on $L^{2}(X)$. Moreover, the maps

$$
\mathscr{L}(X \times K, 1) \ni u \mapsto u(x, D) \in B\left(L^{2}\right)
$$

and

$$
\mathscr{L}(X \times K, 1) \ni u \mapsto u(D, x) \in B\left(L^{2}\right)
$$

are continuous.

(b) If $u \in \mathscr{L}_{\infty}(X \times K, 1)$ then both $u(x, D)$ and $u(D, x)$ are compact.

Now let $X^{a}$ be a vector space and $\pi^{a}: X \rightarrow X^{a}$ a linear surjection. We can identify the dual of $X^{a}$ with a subspace $K^{a}$ of $K$.

The following lemma follows immediately from Theorem A2.6.

Lemma A2.7. (a) Let $u \in \mathscr{L}\left(X^{a} \times K, X^{a} \times K^{a}, 1\right)$. Then $u\left(\pi^{a} x, D\right)$ and $u\left(D, \pi^{a} x\right)$ are bounded and a-fibered.

(b) Let $u \in \mathscr{L}_{\infty}\left(X^{a} \times K, X^{a} \times K^{a}, 1\right)$. Then $u\left(\pi^{a} x, D\right)$ and $u\left(D, \pi^{a} x\right)$ are a-compact.

Now we are ready to state and prove the result that we use directly in the proof of Lemma 6.2.

Proposition A2.8. Let $\eta$ be slowly varying on $X^{a}$ and $\rho$ be $K^{a}$-slowly varying on $K$. Let $f \in C\left(X^{a}\right)$ and $g \in C(K)$ have the property that $\nabla f \in \mathscr{L}\left(X^{a}, \eta\right)$ and $\nabla g \in \mathscr{L}\left(K, K^{a}, \rho\right)$. Then

$$
\eta\left(\pi^{a} x\right)^{-1}\left[f\left(\pi^{a} x\right), g(D)\right] \rho(D)^{-1}
$$

is a-fibered and bounded on $L^{2}(X)$. If moreover

$$
\nabla f \in \mathscr{L}_{\infty}\left(X^{a}, \eta\right)
$$


and

$$
\nabla g \in \mathscr{L}_{\infty}\left(K, K^{a}, \rho\right)
$$

then (A2.15) is a-compact.

Proof. Set $\xi\left(x^{a}, k\right)=\eta\left(x^{a}\right) \rho(k)$ and $u\left(x^{a}, k\right)=f\left(x^{a}\right) g(k)$. Then $\xi$ is $X^{a} \times$ $K^{a}$-slowly varying on $X^{a} \times K$ and

$$
\nabla_{x^{a}} \cdot \nabla_{k} u \in \mathscr{L}\left(X^{a} \times K, X^{a} \times K^{a}, \xi\right) .
$$

Clearly $u\left(\pi^{a} x, D\right)-u\left(D, \pi^{a} x\right)=\left[f\left(\pi^{a} x\right), g(D)\right]$. But by Proposition A2.5 $u\left(\pi^{a} x, D\right)-u\left(D, \pi^{a} x\right)=v\left(\pi^{a} x, D\right)$ where $v=\int_{0}^{1} d \tau e^{i \tau D_{x^{a}} \cdot D_{k}} i D_{x^{a}} \cdot D_{k} u$. By Theorem A2.2

$$
e^{i \tau D_{x^{a}} \cdot D_{k}} i D_{x^{a}} \cdot D_{k} u \in \mathscr{L}\left(X^{a} \times K, X^{a} \times K^{a}, \xi\right) .
$$

The methods of the proof of Theorem A2.2 also show that $e^{i \tau D_{x^{a}} \cdot D_{k}} u$ depends continuously on $\tau$. Thus

$$
v \in \mathscr{L}\left(X^{a} \times K, X^{a} \times K^{a}, \xi\right) .
$$

By Lemma A2.1 we can choose $\tilde{\eta} \in \mathscr{L}\left(X^{a}, \eta\right)$ and $\tilde{\rho} \in \mathscr{L}(K, \rho)$ such that $\eta \leq \tilde{\eta} \leq c_{1} \eta$ and $\rho \leq \tilde{\rho} \leq c_{2} \rho$. Set $\tilde{v}\left(x^{a}, k\right)=\tilde{\eta}^{-1}\left(x^{a}\right) v\left(x^{a}, k\right) \tilde{\rho}^{-1}(k)$. Clearly $\tilde{v} \in \mathscr{L}\left(X^{a} \times K, X^{a} \times K^{a}, 1\right)$. But (A2.15) equals

$$
\left[\eta^{-1}\left(\pi^{a} x\right) \tilde{\eta}\left(\pi^{a} x\right)\right]\left[\tilde{v}\left(\pi^{a} x, D\right)\right]\left[\tilde{\rho}(D) \rho^{-1}(D)\right] .
$$

All the factors in (A2.19) are bounded. Thus (A2.15) is bounded. If moreover (A2.16) and (A2.17) are true then $\tilde{v} \in \mathscr{L}_{\infty}\left(X^{a} \times K, X^{a} \times K^{a}, 1\right)$. Thus by Lemma A2.7(b) the operator (A2.15) is a-compact. Q.E.D.

Proof of Lemma 6.2. We apply Proposition A2.8. We put $\eta\left(x^{a}\right)=1, \rho(k)=$ $\omega(k)+1$ and $f\left(x^{a}\right)=\chi_{b}^{a}\left(x^{a}\right)$. In (a) we set $g(k)=\omega(k)$ and in (b) we set $g(k)=k \cdot \nabla \omega(k)$. Q.E.D.

\section{APPENDIX 3}

In this appendix we sketch the proof of certain simple facts about estimating functions by continuous functions.

Let $f$ be a real function on $\mathbf{R}^{n}$ and $\kappa \leq 0$. Define

$$
f^{\kappa}(x)=\sup _{\kappa^{\prime}>\kappa}\left(\inf _{|x-y| \leq \kappa^{\prime}} f(y)\right) \text {. }
$$

It is easy to show the following properties of the operation $f \mapsto f^{\kappa}$.

Lemma A3.1. (a) $f^{\kappa}$ is measurable (even if $f$ is not).

(b) $\left(f^{\kappa_{1}}\right)^{\kappa_{2}}=f^{\kappa_{1}+\kappa_{2}}$.

(c) Let $j \in C_{0}\left(\mathbb{R}^{n}\right), \kappa_{1}>\kappa_{2}>0$, supp $j \subset B\left(0, \kappa_{1}-\kappa_{2}\right), \int j(x) d x=1$ and $j \geq 0$. Then $f^{\kappa_{1}} \leq f^{\kappa_{2}} * j$ and $f^{\kappa_{1}} * j \leq f^{\kappa_{2}}$.

The following fact is used in $\S 6$. 
Proposition A3.2. Let $\kappa_{1}>\kappa_{2}>0$. Then we can find a continuous function $\xi$ such that $f^{\kappa_{1}} \leq \xi \leq f^{\kappa_{2}}$. If $f$ is bounded then $\xi$ is bounded too.

Proof. Let $\kappa^{\prime}=\left(\kappa_{1}+\kappa_{2}\right) / 2$ and $\kappa^{\prime \prime}=\left(\kappa_{1}-\kappa_{2}\right) / 2$. Let $j \in C_{0}\left(\mathbf{R}^{n}\right)$ such that $\operatorname{supp} j \subset B\left(0, \kappa^{\prime \prime}\right), \int j(x) d x=1$ and $j \geq 0$. We put $\xi=f^{\kappa^{\prime}} * j$. Now we apply Lemma A3.1(c). Q.E.D.

Acknowledgements. I am very grateful to I. Sigal for helpful conversations and for bringing my attention to the subject of dispersive Schrödinger $N$-body operators. I am also indebted to Professor S. L. Woronowicz for an outline of the proof of Lemma A1.1.

\section{REFERENCES}

[M1] E. Mourre, Absence of singular continuous spectrum for certain selfadjoint operators, Comm. Math. Phys. 78 (1981), 391-408.

[PSS] P. Perry, I. M. Sigal and B. Simon, Spectral analysis of $N$-body Schrödinger operators, Ann. of Math. 114 (1981), 519-567.

[FH1] R. Froese and I. Herbst, A new proof of the Mourre estimate, Duke Math. J. 49 (1982), 1075-1085.

[M2] E. Mourre, Opérateurs conjugués et propriétés de propagations, Comm. Math. Phys. 91 (1983), 279-300.

[CFKS] H. L. Cycon, R. Froese, W. Kirsch and B. Simon, Schrödinger operators with application to quantum mechanics and global geometry, Springer-Verlag, Berlin, Heidelberg and New York, 1987.

[Ya] D. R. Yafaev, Remarks on the spectral theory for Schrödinger operators of multiparticle type, Zap. Nauchn. Sem. Leningrad Otdel. Mat. Inst. Steklov. (LOMI) 133 (1984), 277-298. (Russian)

[FH2] R. Froese and I. Herbst, Exponential bounds and absence of positive eigenvalues for $N$-body Schrödinger operators, Comm. Math. Phys. 87 (1982), 429-447.

[SigSof1] I. Sigal and A. Soffer, N-particle scattering problem: Asymptotic completeness for short range systems, Ann. of Math. 125 (1987), 35-108.

[SigSof2] _ private communication.

[A] S. Agmon, Lectures on the exponential decay of solutions of second order elliptic equations, Princeton Univ. Press, Princeton, N.J., 1982.

[RS3] M. Reed and B. Simon, Methods of modern mathematical physics. III, Academic Press, New York, 1979.

[RS4] _ _ Methods of modern mathematical physics. IV, Academic Press, New York, 1978.

[Sig1] I. M. Sigal, Scattering theory for many body quantum mechanical systems, Lecture Notes in Math., vol. 1011, Springer-Verlag, Berlin and New York, 1983.

[Ha] G. A. Hagedorn, Asymptotic completeness for two, three, and four particle Schrödinger operators, Trans. Amer. Math. Soc. 258 (1980), 1-75.

[Ka1] T. Kato, Wave operators and similarity for some non-selfadjoint operators, Math. Ann. 162 (1966), 258-279.

[Ka2] _ Smooth operators and commutators, Studia Math. 31 (1968), 535-546.

[De1] J. Dereziński, $A$ new proof of the propagation theorem for $N$-body quantum systems, preprint, 1988.

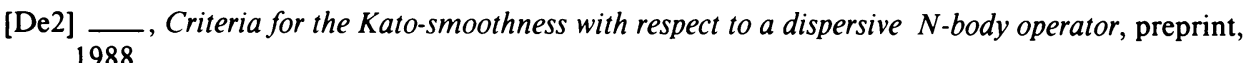
1988. 
[Ka3] T. Kato, Perturbation theory for linear operators (2nd ed.), Springer-Verlag, Berlin, Heidelberg, New York, 1980.

[Kalf] $\mathrm{H}$. Kalf, The quantum mechanical virial theorem and the absence of positive energy bound states of Schrödinger operators, Israel J. Math. 20 (1975), 57-69.

[We] J. Weidmann, The virial theorem and its application to the spectral theory of Schrödinger operators, Bull. Amer. Math. Soc. 73 (1967).

[E] V. Enss, A note on Hunziker's theorem, Comm. Math. Phys. 52 (1977), 233-238.

[DS] P. Deift and B. Simon, A time-dependent approach to the completeness of multiparticle quantum scattering, Comm. Pure Appl. Math. 30 (1977), 573-583.

[Sim] B. Simon, Geometric methods in multiparticle quantum systems, Comm. Math. Phys. 55 (1977), 259-274.

[Sig2] I. M. Sigal, Geometric methods in quantum many body problem. Nonexistence of very negative ions, Comm. Math. Phys. 85 (1982), 309-324.

[JP] A. Jensen and P. Perry, Commutator methods and Besov space estimates for Schrödinger operators, J. Operator Theory 14 (1985), 181-188.

[JPM] A. Jensen, P. Perry and E. Mourre, Multiple commutator estimates and resolvent smoothness in quantum scattering theory, Ann. Inst. H. Poincare Phys. Théor. 41 (1984), 207-225.

[Hö] L. Hörmander, The analysis of linear partial differential operators. III, Springer-Verlag, Berlin, Heidelberg and New York, 1985.

[Ta] M. Taylor, Pseudodifferential operators, Princeton Univ. Press, Princeton, N.J., 1981.

Center for Transport Theory and Mathematical Physics, Virginia Polytechnic InStitute and State University, Blacksburg, Virginia 24061

Current address: Division of Mathematical Methods in Physics, Warsaw University, Hoza 74, 00-682 Warsaw, Poland 